\title{
Extracorporeal shock wave as adjuvant therapy for wrist and hand spasticity in post-stroke patients: a randomized controlled trial
}

\author{
Samar Abd Alhamed Tabra* (D, Mohammad Ibrahim Zaghloul and Doaa Shawky Alashkar
}

\begin{abstract}
Background: Stroke patients often present with upper limb spasticity which impairs the functional status of patients. Recently, extracorporeal shock wave therapy (ESWT) is reported to be a safe, non-invasive, alternative treatment for spasticity. Many articles have been published on the effect of ESWT on lower limb spasticity, but only few of them had focused on upper limb spasticity, so the aim of this study is to evaluate the clinical and electrophysiological effect of ESWT on wrist and hand spasticity of chronic stroke patients and its impact on functional performance. In this monocentric study, forty chronic stroke patients with upper limb spasticity were recruited and randomly allocated into two groups. Both groups continued to receive conventional stroke rehabilitative program, while group I received three sessions of radial extracorporeal shock wave therapy (rESWT) 1 week apart.

Results: There was a significant decrease in wrist and hand spasticity after treatment and at follow-up in group I compared to group II (Modified Ashworth Scale after rESWT 1.45 \pm 0.16, $2.90 \pm 0.18$ and follow-up 1.55 $\pm 0.13,3.00 \pm$ .0 .15 in groups I and II, respectively). Also, there was a significant improvement of wrist control and hand function after treatment and at follow-up in group I compared to group II $(p<0.001)$. The improvement of pinch grip was noticed at follow-up with a significant difference relative to baseline in group I $(p<0.05)$. Hmax/Mmax ratio was significantly decreased at follow-up in group I compared to group II $(p<0.001)$.
\end{abstract}

Conclusion: ESWT is a valuable adjuvant treatment for spasticity of the hand and wrist in stroke patients which is reflected as improvement of functional activity.

Trial registration: ClinicalTrials.gov, NCT04312581. Registered on 18 March 2020.

Keywords: Extracorporeal shock wave therapy, Rehabilitation, Spasticity, Stroke, Upper limb

\section{Background}

Spasticity is defined as "a velocity-dependent enhancement in muscle tone in response to passive stretching because of supraspinal disinhibition of stretch reflexes." Post-stroke upper limb spasticity can be disabling and can result in some functional limitations, representing an example of maladaptive plasticity. The incidence of

\footnotetext{
* Correspondence: dr_stabra_113@yahoo.com

Rheumatology and Rehabilitation Department, Faculty of Medicine, Tanta University, El-Geish Street, Tanta, Gharbia 31527, Egypt
}

post-stroke spasticity is variable ranging from 20 to $40 \%$ and associated with a significant impact on patient's functional status and quality of life [1]. Persistent pain, reduced mobility, contractures, and skeletal deformities are the drawbacks of spasticity that may limit the potential effect of rehabilitation. Therapeutic regimens for spasticity management depend on passive stretching, splints, antispastic drug, phenol, botulinum toxin (BTX) injection, physical modalities as electric therapy and ultrasound therapy, and surgery [2].

\section{Springer Open}

(0) The Author(s). 2021 Open Access This article is licensed under a Creative Commons Attribution 4.0 International License, which permits use, sharing, adaptation, distribution and reproduction in any medium or format, as long as you give appropriate credit to the original author(s) and the source, provide a link to the Creative Commons licence, and indicate if changes were made. The images or other third party material in this article are included in the article's Creative Commons licence, unless indicated otherwise in a credit line to the material. If material is not included in the article's Creative Commons licence and your intended use is not permitted by statutory regulation or exceeds the permitted use, you will need to obtain permission directly from the copyright holder. To view a copy of this licence, visit http://creativecommons.org/licenses/by/4.0/. 
Extracorporeal shock wave therapy (ESWT) is defined as "a sequence of single, highly energetic, biphasic acoustic impulses characterized by rapid propagation of suddenly increased pressure in three-dimensional space." Two types of ESWT are defined, focused (fESWT) and radial (rESWT); focused ESWT (fESWT) is generated by electromagnetic, electrohydraulic, and piezoelectric sources. In fESWT, the pressure increases rapidly from under $10 \mathrm{~ns}$ up to 100-1000 bars (energy absorption up to $12-\mathrm{cm}$ depth). In the radial ESWT (rESWT), the pressure increases slightly up to $5 \mu$ s and reaching $1-10$ bars (energy absorption up to $3 \mathrm{~cm}$ ). So fESWT is more intensive than rESWT within the focal area of the highest energy exposure when rESWT has the superficial region of interest. The rESWT is less invasive than fESWT and more appropriate for physiotherapy purposes [3].

ESWT has been successfully used in orthopedic diseases such as tendinitis, epicondylitis, plantar fasciitis, and several inflammatory tendon diseases [4].

In the last 5 years, a few review studies have provided evidence to support the use of ESWT for the spasticity: one meta-analysis of clinical trials on all types of spasticity in patients after brain injury [5], two meta-analyses of randomized controlled trials (RCTs) on spasticity in post-stroke patients $[2,6]$, and one authorized narrative review on upper and lower limb spasticity in post-stroke patients [7]. Several studies have confirmed the use of ESWT in decreasing spasticity in patients with cerebral palsy [8-13] and multiple sclerosis [14].

The underlying mechanisms that explain the beneficial effects of ESWT on spasticity are still undefined. Previous studies have suggested the effect of ESWT on nitric oxides (NO) production [15], muscle fibrosis [16], spinal cord excitability modification [17], or affect the Golgi tendon directly which suppresses motor nerve excitability by inhibiting muscle spindle activity [18].

As many articles that have been published on the effect of ESWT were performed on the lower limb spasticity $[8-10,12-14,19-21]$, only a few of them had focused on upper limb spasticity [22]. We aimed to evaluate the clinical and electrophysiological effect of extracorporeal shock wave therapy (ESWT) on the wrist and hand spasticity of chronic stroke patients and its impact on functional performance.

\section{Methods}

\section{Study design}

This is a prospective, single-center, double-blind randomized controlled parallel-group trial. Ethical approval for this study was obtained from the ethics committee of Tanta. University Faculty of Medicine (approval number: 33693/2/20).

\section{Study setting}

Patients were selected from the outpatient clinic of Physical Medicine, Rheumatology and Rehabilitation Department of Tanta University Hospitals, Egypt.

\section{Participant}

This study was carried out on forty chronic stroke patients who presented at the time of study with upper limb spasticity at different degrees. The sample size estimation was performed using the G-Power 3.1.9.2 software. It was found that 11 individuals for each group must have been recruited to have $85 \%$ power with $5 \%$ type 1 error level. The average expected value in the first group was -0.5 (with a standard deviation of 0.09 ), and the average expected value in the second group was 0.05 (with a standard deviation of 0.05 ) based on the previous research conducted by $\mathrm{Li}$ et al. evaluating the effect of radial shock wave therapy on spasticity of the upper limb in patients with chronic stroke.

Informed written consent from all patients was obtained in accordance with the local ethical committee. Privacy of all patients' data was granted as there was a code number for every patient file.

\section{Inclusion criteria}

Patients with chronic stroke with a disease duration of more than 1 year were included in the study with a stable Modified Ashworth Scale for upper limb spasticity ranged from $1+$ to 4 .

\section{Exclusion criteria}

Patients more than 65 years (to limit the effect of age on muscle bulk and power); patients with double stroke; patients with fixed contractures of the wrist and hand; patients who had received antispastic measures (botulinum toxins, nerve block) within 6 months; patients with contraindication to extracorporeal shock wave therapy, i.e., malignancy at the treatment area, coagulopathies, and active infection (viral or TB); patients with oral anticoagulants and bleeding wounds; and patients with pacemakers.

\section{Randomization}

The patients were randomly divided into two groups (20 patients for each) by using a computer-generated random number of sequences. The group assignment was recorded on a card. This card was folded in half such that the label with the patient's group assignment was on the inside of the fold. The folded card was then placed inside the envelope, and the envelope was sealed. 


\section{Interventions \\ First group}

The first group received three sessions of radial extracorporeal shock wave therapy (rESWT) 1 week apart, 2000-3000 impulses at $0.25-0.84 \mathrm{~mJ} / \mathrm{mm}^{2}$ with a pressure $2.8 \mathrm{bar}$ and $15 \mathrm{~Hz}$ frequency. rESWT was applied on flexor carpi ulnaris, flexor carpi radialis, flexor digitorum muscles nearly at the midpoint of their fleshy part, intrinsic hand muscles, and tendons of flexor digitorum on the palm. The patients continued their medical treatment and conventional rehabilitation program.

\section{Second group}

The second group received conventional rehabilitation in form of range of motion exercise of upper limb joints; passive stretching exercise for fingers, wrist, and elbow flexors; and occupational therapy, in addition to medical treatment for spasticity management. The patients in both groups received the same conventional rehabilitation program: three sessions/week (45 min each session) for the whole period of the study till the time of followup.

\section{Outcome measurements}

Patients were examined by the same physiatrist, who was blinded to the randomization and treatment procedure. The evaluation was performed at the baseline, 2 weeks, and 3 months after the last session of rESWT as a follow-up.

\section{Modified Ashworth Scale (MAS) [23]}

Scoring (taken from Bohannon and Smith [23]) is a valid scoring system for spasticity; it consists of six grades $\{0$ no increase in muscle tone; 1 slight increase in muscle tone, manifested by a catch and release or by minimal resistance at the end of the range of motion when the affected part(s) is moved in flexion or extension; 1+ slight increase in muscle tone, manifested by a catch, followed by minimal resistance throughout the remainder (less than half) of the range of motion (ROM); 2 more marked increase in muscle tone through most of the ROM, but affected part(s) easily moved; 3 considerable increase in muscle tone, passive movement difficult; 4 affected part(s) rigid in flexion or extension\}. Grade 1+ was pointed as 2 in the study; therefore, 1 point was added to each of the following grades, so the scale was pointed from 0 to 5 . The patient was supine with the elbow as straight as possible and the wrist was moved passively from flexion to extension.

\section{Fugl-Meyer Assessment (FMA) [24]}

The FMA assesses motor function recovery after stroke and consists of 33 and 17 performance items in the upper and lower limbs, respectively. The scores range from 0 (unable to perform), 1 (partial ability to perform), to 2 (near-normal ability to perform). The items that measure wrist control and hand function have been revealed to have excellent intrarater reliability and high interrater reliability [25].

\section{Motricity Index [26]}

The Motricity Index can be used to assess the motor impairment in a patient with stroke; only one item (pinch grip) was tested using a $2.5-\mathrm{cm}$ cube between the thumb and forefinger and the score was graded as:

- 0 no movement

- 11 beginnings of prehension (any movement of fingers or thumb)

- 19 able to grip cube but not hold it against gravity

- 22 able to hold cube against gravity but not against a weak pull

- 26 able to hold the cube against a weak pull but strength is weaker than normal

- 33 normal pinch grip

The validity of the Motricity Index for the upper extremity is supported by the high degree of association between its components and its correlation with both grip strength and a measure of upper extremity function [27].

\section{Electrophysiological assessment of spasticity by Hmax/ Mmax amplitude ratio}

Nihon Kohden neuropack 2 electromyography (EMG) machine, 2 channel, surface electrodes were used. $\mathrm{H}$ reflexes occur consistently in a variety of upper extremity muscles during an isometric contraction. Hmax/Mmax amplitude ratio was obtained from the patient's spastic upper limb. Flexor carpi radialis muscle (FCR) which was the recording site with active electrode placement was placed at a point between the proximal one-fourth and the distal three-fourths (recording point) on a line between the medial epicondyle of the humerus and the styloid process of the radius, reference over its tendon. The ground electrode was put between the recording electrode and the stimulator. Submaximal stimulation was done for the median nerve between the biceps brachii and brachialis muscles at the elbow using a bipolar stimulator with the cathode proximal to the anode [28]. After that, supramaximal stimulation was done (at the same previous site of stimulation with the cathode distal to the anode) to produce the maximal direct muscle (M) response from FCR. The $\mathrm{H} / \mathrm{M}$ ratio was calculated as each value is a mean of three consecutive values of both $\mathrm{H}$ reflex and $\mathrm{M}$ response. The Hmax/Mmax ratio is a reliable measure of the $\alpha$ motor neuron excitability [29]. 


\section{Statistical analysis}

Data were fed to the computer and analyzed using IBM SSPS software package version 20.0 (Armonk, NY: IBM Corp.). Qualitative data were described using number and percent, and quantitative data were described using range, mean, standard deviation. The significance of the obtained results was adjusted at the $5 \%$ level.

A paired $t$ test was used for normally distributed quantitative variables, to compare between two periods, and a Student $t$ test to compare between two studied groups. Statistical significance was set at $p \leq 0.05$ [30].

\section{Results}

\section{Participants}

The flow diagram of the patients who participated in this study is shown in Fig. 1. The demographic and clinical data of patients are listed in Table 1. Both groups showed no significant difference regarding Modified Ashworth Scale (MAS) for wrist and hand muscles,
Fugl-Myer Assessment (FMA) for wrist control and hand function, and Motricity Index at the baseline.

\section{Outcome measurements}

There was a significant improvement of MAS and FMA at 2 weeks after rESWT and at 3-month follow-up in the first group compared to the second. Also, there was a significant improvement of MAS and FMA in patients of the first group after treatment and at follow-up relative to baseline, but there was no significant difference at followup compared to after treatment except for hand function of FMA. Patients of the second group showed no significant change at the two periods (Tables 2 and 3 ).

Regarding Motricity Index for pinch grip, there was no significant difference between patients of both groups after treatment and at follow-up; however, the only significant improvement of this index was recorded at the follow-up period when compared to baseline in the first group (Table 3).

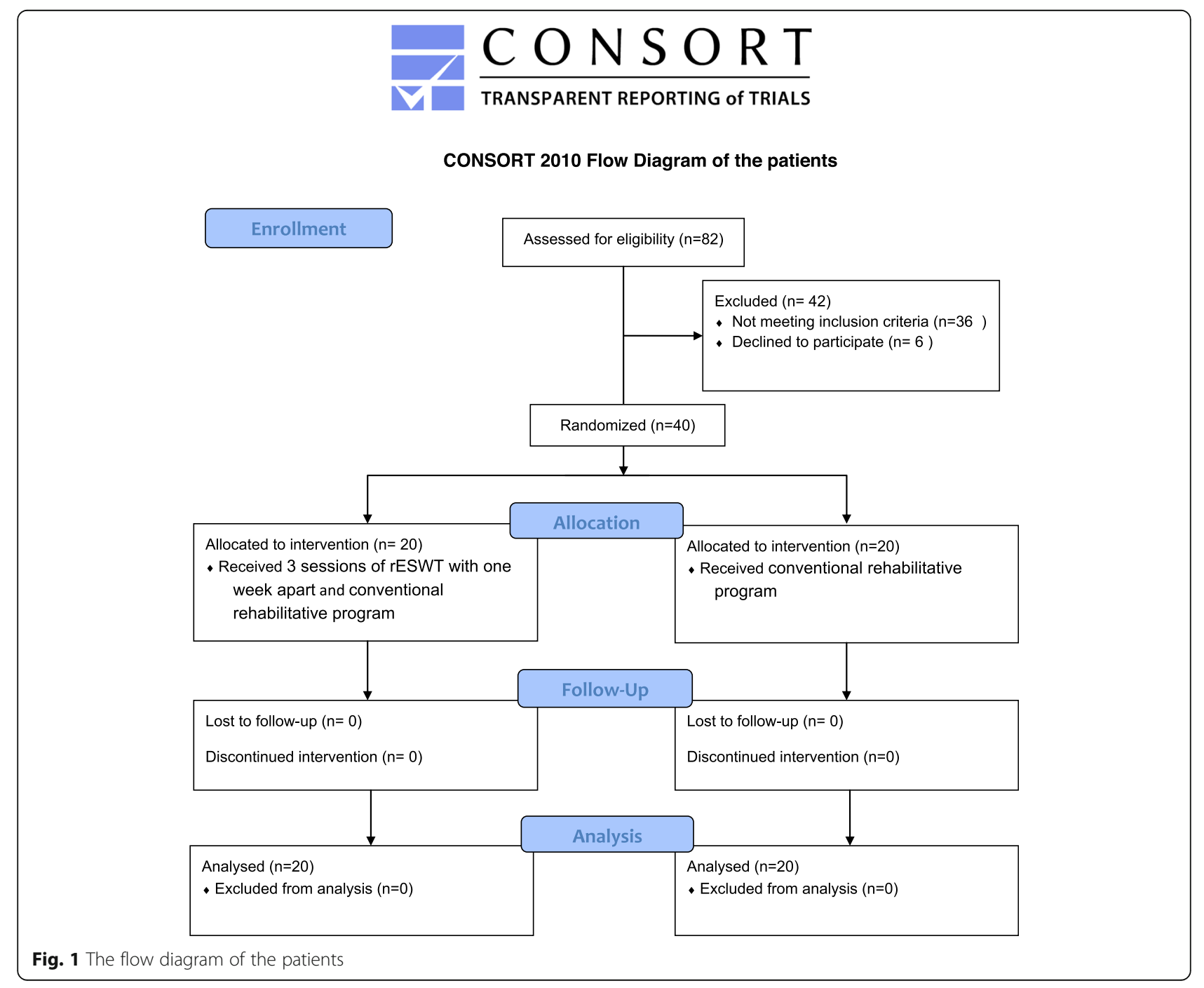


Table 1 Demographic and clinical data of the studied groups showing that the two groups are matched regarding patient's age and disease duration

\begin{tabular}{|c|c|c|c|c|c|}
\hline & \multicolumn{2}{|c|}{ Group I $(n=20)$} & \multicolumn{2}{|c|}{ Group II $(n=20)$} & Unpaired $t$ test $(p)$ \\
\hline \multicolumn{6}{|l|}{ Age (years) } \\
\hline Mean \pm SD & \multicolumn{2}{|c|}{$55.70 \pm 9.30$} & \multicolumn{2}{|c|}{$53.85 \pm 10.20$} & 0.557 \\
\hline Sex & No. & $\%$ & No. & $\%$ & - \\
\hline Male & 18 & 90 & 17 & 85 & \\
\hline Female & 2 & 10 & 3 & 15 & \\
\hline Type of stroke & No. & $\%$ & No. & $\%$ & - \\
\hline Ischemic & 13 & 65 & 11 & 55 & \\
\hline Hemorrhagic & 7 & 35 & 9 & 45 & \\
\hline Side affected & No. & $\%$ & No. & $\%$ & - \\
\hline Right & 15 & 75 & 12 & 60 & \\
\hline Left & 5 & 25 & 8 & 40 & \\
\hline \multicolumn{6}{|c|}{ Disease duration (months) } \\
\hline Mean \pm SD & \multicolumn{2}{|c|}{$33.50 \pm 5.60$} & \multicolumn{2}{|c|}{$31.70 \pm 9.15$} & 0.462 \\
\hline
\end{tabular}

Group I, radial extracorporeal shock wave therapy and conventional rehabilitation; group II, conventional rehabilitation

Hmax/Mmax ratio was significantly decreased at follow-up in patients of the first group (Fig. 2) compared to the second but not after treatment. Also, this ratio was significantly decreased after the treatment and at follow-up in the first group (Table 4).

Figure 2 shows $\mathrm{H}$ reflex in group I before treatment, 2 weeks after treatment, and at follow-up with $\mathrm{H} / \mathrm{M}$ ratio
0.84, 0.67 , and 0.40 before treatment, 2 weeks after treatment, and at follow-up, respectively.

No adverse events were observed in either group.

\section{Discussion}

The present study suggests that rESWT has a beneficial effect on spasticity and functional status of the upper

Table 2 Modified Ashworth Scale (MAS) for the wrist and hand in the studied groups at baseline, after rESWT, and at follow-up showing significant improvement in Gl after receiving rESWT therapy relative to baseline and also a significant improvement in comparison to Gll

\begin{tabular}{llll}
\hline & $\begin{array}{l}\text { Group I } \\
\text { Mean } \pm \text { SD }\end{array}$ & $\begin{array}{l}\text { Group II } \\
\text { Mean } \pm \text { SD }\end{array}$ & Unpaired $t$ test $(p)$ \\
\hline MAS (wrist) & & & 0.094 \\
Baseline & $3.05 \pm 0.18$ & $2.95 \pm 0.19$ & $<0.001^{*}$ \\
After rESWT (2 weeks after the last session) & $1.45 \pm 0.16$ & $2.90 \pm 0.18$ & $<0.001^{*}$ \\
Follow-up (3 months after the last session) & $1.55 \pm 0.13$ & $3.00 \pm .0 .15$ & \\
Paired $\boldsymbol{t}$ test & & 0.396 & 0.064 \\
P1 & $<0.001^{*}$ & 0.361 & \\
P2 & $<0.001^{*}$ & & $<.145$ \\
P3 & 0.372 & $3.25 \pm 0.21$ \\
MAS (hand) & & $3.15 \pm 0.18$ \\
Baseline & $3.35 \pm 0.22$ & $3.20 \pm 0.16$ \\
After rESWT (2 weeks after last session) & $2.15 \pm 0.19$ & \\
Follow-up (3 months after the last session) & $2.20 \pm 0.17$ & 0.107 \\
Paired $\boldsymbol{t}$ test & & 0.359 \\
P1 & $<0.001^{*}$ & 0.391 \\
P2 & $<0.001^{*}$ & $0.001^{*}$ \\
P3 & 0.388 & \\
\hline
\end{tabular}

Values are mean \pm SD

MAS Modified Ashworth Scale, P1 2 weeks after rESWT versus baseline, P2 3-month follow-up versus baseline, P3 3-month follow-up versus 2 weeks after rESWT *Significant values 
Table 3 Fugl-Myer Assessment (FMA) of hand function and wrist control and Motricity Index (pinch grip) in the studied groups at baseline, after rESWT, and at follow-up showing significant improvement of FMA in Gl after receiving rESWT therapy relative to baseline and in comparison to Gll and significant improvement of Motricity Index at follow-up period in Gl

\begin{tabular}{|c|c|c|c|}
\hline & $\begin{array}{l}\text { Group I } \\
\text { Mean } \pm \text { SD }\end{array}$ & $\begin{array}{l}\text { Group II } \\
\text { Mean } \pm \text { SD }\end{array}$ & Unpaired $t$ test $(p)$ \\
\hline \multicolumn{4}{|l|}{ FMA (wrist control) } \\
\hline Baseline & $1.20 \pm 0.17$ & $1.25 \pm 0.23$ & 0.436 \\
\hline After rESWT ( 2 weeks after the last session) & $2.10 \pm 0.35$ & $1.30 \pm 0.21$ & $<0.001^{*}$ \\
\hline Follow-up ( 3 months after the last session) & $2.00 \pm 0.16$ & $1.35 \pm 0.19$ & $<0.001^{*}$ \\
\hline \multicolumn{4}{|l|}{ Paired $t$ test } \\
\hline P1 & $<0.001^{*}$ & 0.476 & \\
\hline P2 & $<0.001^{*}$ & 0.140 & \\
\hline P3 & 0.266 & 0.439 & \\
\hline \multicolumn{4}{|l|}{ FMA (hand function) } \\
\hline Baseline & $3.65 \pm 0.24$ & $3.75 \pm 0.32$ & 0.272 \\
\hline After rESWT ( 2 weeks after the last session) & $5.20 \pm 0.36$ & $3.70 \pm 0.34$ & $<0.001^{*}$ \\
\hline Follow-up ( 3 months after the last session) & $4.70 \pm 0.18$ & $3.80 \pm 0.28$ & $<0.001^{*}$ \\
\hline \multicolumn{4}{|l|}{ Paired $t$ test } \\
\hline P1 & $<0.001^{*}$ & 0.632 & \\
\hline P2 & $<0.001^{*}$ & 0.600 & \\
\hline P3 & $<0.001^{*}$ & 0.313 & \\
\hline \multicolumn{4}{|l|}{ Motricity Index (pinch grip) } \\
\hline Baseline & $20.45 \pm 3.37$ & $21.05 \pm 2.48$ & 0.521 \\
\hline After rESWT ( 2 weeks after the last session) & $22.70 \pm 4.10$ & $21.40 \pm 3.23$ & 0.272 \\
\hline Follow-up ( 3 months after the last session) & $23.15 \pm 4.62$ & $21.85 \pm .3 .70$ & 0.336 \\
\hline \multicolumn{4}{|l|}{ Paired $t$ test } \\
\hline P1 & 0.064 & 0.707 & \\
\hline P2 & $0.043^{*}$ & 0.432 & \\
\hline P3 & 0.747 & 0.682 & \\
\hline
\end{tabular}

Values are mean $\pm \mathrm{SD}$

FMA Fugl-Meyer Assessment, P1 2 weeks after rESWT versus baseline, P2 3-month follow-up versus baseline, P3 3-month follow-up versus 2 weeks after rESWT *Significant values

limb (using Fugl-Meyer Assessment for hand function and wrist control and Motricity Index for pinch grip).

Neuronal plasticity can lead to an extreme degree of spontaneous recovery of early stroke patients in few months. However, neural plasticity concerning compensatory movement, activated ipsilateral motor projections, and competitive interaction after stroke contributes to maladaptive plasticity, which negatively affects motor recovery. Rehabilitation programs should be selected according to the motor impairment of stroke patients to minimize maladaptive plasticity [31]. We conducted this study on patients with a disease duration of more than 1 year aiming to investigate the individual effect of treatment regimens on spastic muscles.

Despite no established standard guidelines, several mechanisms could explain the therapeutic role of ESWT in spasticity; nitric oxides (NO) generated by ESWT are involved in neurotransmission, memory formation, neuromuscular junctions, and synaptic plasticity in the central nervous system [4]. Similar to the inhibitory action of botox (BTX) on neuromuscular transmission, Kenmoku et al. observed rapid degeneration of acetylcholine receptors after ESWT application but without muscle weakness. Also, the amplitude of the compound muscle action potential was significantly decreased immediately after ESWT and lasted for 8 weeks in an animal study [32].

Modification of spinal excitability is another mechanism; as abnormal stretch reflexes may not completely explain the development of spasticity, the effects of ESWT on spinal excitability may support the idea that ESWT acts on nonreflex hypertonia [33]. Although having a temporary effect, mechanical vibration could be attributed as a contributing factor decreasing spinal excitability [16]. Structural and mechanical changes in the spastic muscle lead to fibrosis of inactive connective 


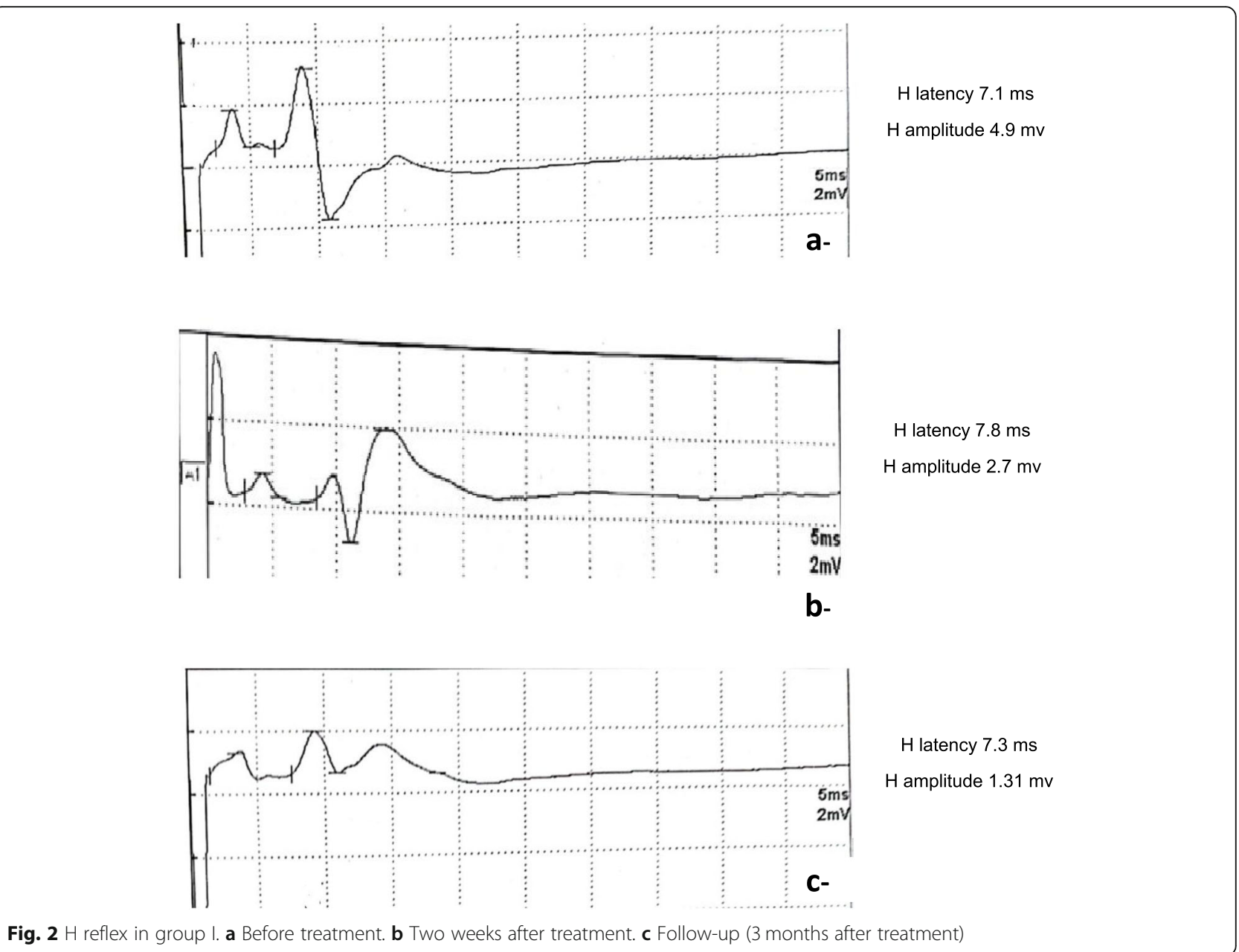

tissue. ESWT in the long run can diminish spasticity by reduction of fibrosis of chronic hypertonic muscles $[2,16]$.

Dymarek et al. [34] revealed a significant reduction in MAS in 20 patients with upper limb spasticity treated with a single session of rESWT. Li et al. [35] reported a significant reduction in spasticity of the hand and wrist for at least 16 and 8 weeks in the group receiving 3 sessions of rESWT and in the group receiving a single session of rESWT, respectively. Three sessions of rESWT had a longer-lasting effect than one session.

Table 4 Hmax/Mmax ratio in the studied groups at baseline, after rESWT, and at follow-up showing a significant decrease of Hmax/ Mmax ratio in Gl after rESWT therapy relative to baseline and significant improvement in comparison to Gll at follow-up

\begin{tabular}{llll}
\hline & $\begin{array}{l}\text { Group I } \\
\text { Mean } \pm \text { SD }\end{array}$ & $\begin{array}{l}\text { Group II } \\
\text { Mean } \pm \text { SD }\end{array}$ & Unpaired $t$ test $(p)$ \\
\hline Hmax/Mmax ratio & & & 0.735 \\
Baseline & $0.79 \pm 0.14$ & $0.77 \pm 0.22$ & 0.283 \\
After rESWT (2 weeks after the last session) & $0.71 \pm 0.10$ & $0.75 \pm 0.23$ & $0.0014^{*}$ \\
Follow-up (3 months after the last session) & $0.58 \pm 0.12$ & $0.76 \pm .0 .19$ & \\
Paired $t$ test & & 0.727 & 0.879 \\
P1 & $0.048^{*}$ & 0.847 \\
P2 & $<0.001^{*}$ & $0.006^{*}$ & \\
P3 & &
\end{tabular}

Values are mean \pm SD

P1 2 weeks after rESWT versus baseline, P2 3-month follow-up versus baseline, P3 3-month follow-up versus 2 weeks after rESWT *Significant values 
Coinciding with the previous data [34, 35], MAS for clinical assessment of the wrist and hand muscle spasticity in stroke patients pointed out significant improvement after treatment and at follow-up compared to baseline in the first group with a significant difference between the two groups (Table 2).

FMA for wrist control and hand function was our tool to assess the sensorimotor function of the wrist and hand in this study; improvement of functional ability was noticed in the first group relative to the second one after treatment and at follow-up with significant differences. Also, there was a significant improvement in patients of the first group after treatment and at follow-up relative to baseline.

Spastic hand muscles may serve as a reasonable factor for an average hand grip more than weak muscles; this can explain the results of the Motricity Index for pinch grip after treatment, where there was no significant change after treatment and at follow-up between both groups, with significant improvement at follow-up period when compared to baseline in the first group.

Few studies measured the motor functional outcome of the upper limb in spastic stroke patients. Troncati et al. [36] revealed significant improvement of FMA of twelve patients with stroke after treatment with two sessions of ESWT, and the effects were maintained 3 and 6 months after treatment.

In a prospective randomized, single-blind controlled trial by $\mathrm{Li}$ et al. [35], a significant improvement in FMA scores for hand function and wrist control after three sessions of rESWT was maintained for 16 and 12 weeks, respectively, compared with those of sham or one session of rESWT group and there was no significant improvement in FMA scores after one session of rESWT compared with sham control, indicating that repeated sessions of ESWT are necessary to ameliorate functional motricity. Thus, clinical experience indicates repeated sessions of ESWT could be superior to a single application. Li et al. [35] confirmed that repeated sessions of rESWT result in a more noticeable and longer-lasting effect.

In 1974, Deschuytere and colleagues [37] showed resting $\mathrm{H}$ reflexes in the flexor carpi radialis (FCR) and palmaris longus in most of their volunteers. $\mathrm{H}$ reflex amplitudes are increased with respect to maximal $M$ responses recorded in the spastic muscle as a result of enhanced excitability in the monosynaptic stretch reflex arc [38].

Our results revealed a significant reduction in Hmax/ Mmax amplitude ratio at follow-up in the first group with a significant difference between the two groups at follow-up (Table 4); these results pointed out the delayed effect of ESWT on reflex arc excitability.

The effect of ESWT on spinal excitability and Golgi tendon organs to suppress motor nerve excitability can be a reasonable mechanism. One recent study revealed a reduction of the Hmax/Mmax ratio after ESWT indicating a change in alpha motor neuron excitability [39]. Other studies revealed that no significant changes of $\mathrm{F}$ wave or $\mathrm{H}$ wave latency or amplitude were demonstrated in human studies after ESWT application [40, 41].

To our knowledge, this study is the first one using combined different assessment measures (clinical and electrophysiological) to evaluate the effect of radial extracorporeal shock wave therapy (rESWT) on wrist and hand functions of spastic chronic stroke patients.

Some limitations of this study should be considered as relatively small sample size of patients and short duration of follow-up to reveal the sustained effects of this modality of treatment. More studies are needed with more flexible inclusion criteria for the disease duration to include spastic patients during the first year to prevent the development of maladaptive plasticity. Also, more research should be done to see if the rESWT could substitute other modalities of spasticity treatment such as the muscle relaxant and botox injection.

\section{Conclusions}

ESWT is a valuable adjuvant treatment for spasticity of the hand and wrist in stroke patients which is reflected as improvement of functional activity.

\section{Recommendation}

rESWT is recommended as adjuvant physical treatment if the muscle relaxants failed to achieve the desired effect or had side effects or contraindications.

\section{Abbreviations}

rESWT: Radial extracorporeal shock wave therapy; BTX: Botulinum toxin; fESWT: Focused extracorporeal shock wave therapy; RCTs: Randomized controlled trials; NO: Nitric oxides; MAS: Modified Ashworth Scale; ROM: Range of motion; FMA: Fugl-Meyer Assessment

\section{Acknowledgements}

Not applicable.

\section{Authors' contributions}

TSA and ADS designed the study protocol. ZMI, TSA, and ADS monitored the progress and collected the data. ADS did the statistical analysis. All authors contributed to drafting the manuscript and have read and approved the final version of the manuscript.

\section{Funding}

The authors received no financial support for the research, authorship, and/ or publication of this article.

Availability of data and materials

The data will be available upon reasonable request.

\section{Declarations}

Ethics approval and consent to participate

Ethical approval for this study was obtained from the Ethics Committee of University faculty of Medicine (approval number: 33693/2/20). Informed written consents from all patients were obtained in accordance with the local ethical committee. 


\section{Consent for publication}

Not applicable.

\section{Competing interests}

The authors declare that they have no competing interests.

\section{Received: 30 December 2020 Accepted: 26 February 2021} Published online: 12 April 2021

\section{References}

1. Watkins CL, Leathley MJ, Gregson JM, Moore AP, Smith TL, Sharma AK (2002) Prevalence of spasticity post stroke. Clin Rehabil 16(5):515-522. https://doi.org/10.1191/0269215502cr512oa

2. Xiang J, Wang W, Jiang W, Qian Q (2018) Effects of extracorporeal shockwave therapy on spasticity in post-stroke patients: a systematic review and meta-analysis of randomized controlled trials. J RehabilMed 50(10):852859

3. Dymarek R, Ptaszkowski K, Ptaszkowska L, Kowal M, Sopel M, Taradaj J, Rosińczuk J (2020) Shock waves as a treatment modality for spasticity reduction and recovery improvement in post-stroke adults-current evidence and qualitative systematic review. Clin Interv Aging 15:9-28. https://doi.org/10.2147/CIA.S221032

4. Mariotto S, de Prati AC, Cavalieri E, Amelio E, Marlinghaus E, Suzuki H (2009) Extracorporeal shock wave therapy in inflammatory diseases: molecular mechanism that triggers anti-inflammatory action. Curr Med Chem 16(19): 2366-2372. https://doi.org/10.2174/092986709788682119

5. Lee J-Y, Kim S-N, Lee I-S, Jung H, Lee K-S, Koh S-E (2014) Effects of extracorporeal shock wave therapy on spasticity in patients after brain injury: a meta-analysis. J Phys Ther Sci 26(10):1641-1647

6. Guo P, Gao F, ZhaoT SW, Wang B, Li Z (2017) Positive effects of extracorporeal shock wave therapy on spasticity in poststroke patients: a meta-analysis. J Stroke Cerebrovasc Dis Off J Natl Stroke Assoc 26(11):24702476. https://doi.org/10.1016/j.jstrokecerebrovasdis.2017.08.019

7. Dymarek R, Ptaszkowski K, Słupska L, Halski T, Taradaj J, Rosińczuk J (2016) Effects of extracorporeal shock wave on upper and lower limb spasticity in post-stroke patients: A narrative review. Top Stroke Rehabil 23(4):293-303. https://doi.org/10.1080/10749357.2016.1141492.

8. Park D-S, Kwon DR, Park G-Y, Lee MY (2015) Therapeutic effect of extracorporeal shock wave therapy according to treatment session on gastrocnemius muscle spasticity in children with spastic cerebral palsy: a pilot study. Ann Rehabil Med 39(6):914-921. https://doi.org/10.5535/arm.201 5.39.6.914

9. Picelli A, Marchina EL, Gajofatto F, Pontillo A, Vangelista A, Filippini R, Baricich A, Cisari C, Smania N (2017) Sonographic and clinical effects of botulinum toxin type A combined with extracorporeal shockwave therapy on spastic muscles of children with cerebral palsy. Dev Neurorehabil 20(3): 160-164

10. Amelio $E$, Manganotti $P$ (2010) Effect of shock wave stimulation on hypertonic plantar flexor muscles in patients with cerebral palsy: a placebocontrolled study. J Rehabil Med 42(4):339-343. https://doi.org/10.2340/1 6501977-0522

11. Vidal X, Morral A, Costa L, Tur M (2011) Radial extracorporeal shock wave therapy (rESWT) in the treatment of spasticity in cerebral palsy: a randomized, placebo-controlled clinical trial. NeuroRehabilitation 29(4):413419. https://doi.org/10.3233/NRE-2011-0720

12. Gonkova MI, Ilieva EM, Ferriero G, Chavdarov I (2013) Effect of radial shock wave therapy on muscle spasticity in children with cerebral palsy. Int J Rehabil Res Int Z Für Rehabil Rev Int Rech Réadapt 36(3):284-290

13. Wang T, Du L, Shan L, Dong H, Feng J, Kiessling MC, Angstman NB, Schmitz C, Jia F (2016) A prospective case-control study of radial extracorporeal shock wave therapy for spastic plantar flexor muscles in very young children with cerebral palsy. Medicine (Baltimore) 95(19):e3649. https://doi. org/10.1097/MD.0000000000003649

14. Marinelli LM (2014) Effect of radial shock wave therapy on pain and muscle hypertonia: a double-blind study in patients with multiple sclerosis. Mult Scler Houndmills Basingstoke Engl 21:5

15. Mariotto S, Cavalieri E, Amelio E, Ciampa AR, Carcereri de Prati A, Marlinghaus E, Russo S, Suzuki H (2005) Extracorporeal shock waves: from lithotripsy to anti-inflammatory action by NO production. Nitric Oxide 12(2): 89-96. https://doi.org/10.1016/j.niox.2004.12.005
16. Manganotti $P$, Amelio E (2005) Long-term effect of shock wave therapy on upper limb hypertonia in patients affected by stroke. Stroke 36(9):19671971. https://doi.org/10.1161/01.STR.0000177880.06663.5c

17. Leone JA, Kukulka CG (1988) Effects of tendon pressure on alpha motor neuron excitability in patients with stroke. Phys Ther 68(4):475-480. https:// doi.org/10.1093/ptj/68.4.475

18. Bae H, Lee JM, Lee KH (2010) The effects of extracorporeal shock wave therapy on spasticity in chronic stroke patients. J Korean Acad Rehabil Med 34:663-669

19. Wu Y-T, Chang C-N, Chen Y-M, Hu G-C (2018) Comparison of the effect of focused and radial extracorporeal shock waves on spastic equinus in patients with stroke: a randomized controlled trial. Eur J Phys Rehabil Med 54(4):518-525. https://doi.org/10.23736/S1973-9087.17.04801-8

20. Taheri P, Vahdatpour B, Mellat M, Ashtari F, Akbari M (2017) Effect of extracorporeal shock wave therapy on lower limb spasticity in stroke patients. Arch Iran Med 20(6):338-343

21. Santamato A, Micello MF, Panza F, Fortunato F, Logroscino G, Picelli A, Manganotti P, Smania N, Fiore P, Ranieri M (2014) Extracorporeal shock wave therapy for the treatment of post stroke plantar-flexor muscles spasticity: a prospective open-label study. Top Stroke Rehabil 21(1):S17-S24. https://doi.org/10.1310/tsr21S1-S17

22. Guoxing, $X$, Zhang, Y, Yinmeng Z, Haoyang D, Ping L, Na L, Fuqian L, Zhenlan $L$ (2018) Therapeutic effect of slow stretching training combined with extracorporeal shock wave on biceps brachii spasticity in stroke patients. J Jilin Univ (Med Ed) 44(2): 374-378.

23. Bohannon R, Smith M (1987) Interrater reliability of a modified Ashworth scale of muscle spasticity. Phys Ther 67(2):206

24. Fugl-Meyer AR, Jaasko L, Leyman I, Olsson S, Steglind S (1975) The poststroke hemiplegic patient. A method for evaluation of physical performance. Scand J Rehabil Med 7(1):13-31

25. Duncan PW, Propst M, Nelson SG (1983) Reliability of the Fugl-Meyer assessment of sensorimotor recovery following cerebrovascular accident. Phys Ther 63(10):1606-1610. https://doi.org/10.1093/ptj/63.10.1606

26. Collin C, Wade D (1990) Assessing motor impairment after stroke: a pilot reliability study. J Neurol Neurosurg Psychiatry 53(7):576-579. https://doi. org/10.1136/jnnp.53.7.576

27. Bohannon RW (1999) Motricity index scores are valid indicators of paretic upper extremity strength following stroke. J Phys Ther Sci 11(2):59-61

28. Zheng C, Zhu Y, Lv F, Ma X, Xia X, Wang L, Jin X, Weber R, Jiang J, Anuvat K (2014) Abnormal flexor carpi radialis H-reflex. J Clin Neurophysiol 31(6):529534

29. Angel RW, Hoffmann WW (1963) The H reflex in normal, spastic and rigid subjects. Arch Neurol 8(6):591-596. https://doi.org/10.1001/archneur.1963. 00460060021002

30. Kirkpatrick LA, Feeney BC (2013) A simple guide to IBM SPSS statistics for version 20.0 student ed. Wadsworth, Cengage Learning, Belmont

31. Takeuchi N, Izumi S (2012) Maladaptive plasticity for motor recovery after stroke: mechanisms and approaches. Neural Plast 2012:359728

32. Kenmoku T, Ochiai N, Ohtori S, Saisu T, Sasho T, Nakagawa K, Iwakura N, Miyagi M, Ishikawa T, Tatsuoka H, Inoue G, Nakamura J, Kishida S, Saito A, Takahashi K (2012) Degeneration and recovery of the neuromuscular junction after application of extracorporeal shock wave therapy. J Orthop Res 30(10):1660-1665. https://doi.org/10.1002/jor.22111

33. Galiana L, Fung J, Kearney R (2005) Identification of intrinsic and reflex ankle stiffness components in stroke patients. Exp Brain Res 165(4):422-434. https://doi.org/10.1007/s00221-005-2320-z

34. Dymarek R, Taradaj J, Rosińczuk J (2016) Extracorporeal shock wave stimulation as alternative treatment modality for wrist and fingers spasticity in poststroke patients: a prospective, open-label, preliminary clinical trial. Evid Based Complement Alternat Med 2016:1-10. https://doi.org/10.1155/2016/4648101

35. Li TY, Chang CY, Chou YC, Chen LC, Chu HY, Chiang SL, Chang ST, Wu YT (2016) Effect of radial shock wave therapy on spasticity of the upper limb in patients with chronic stroke. A prospective, randomized, single blind, controlled trial. Medicine 95(18):e3544

36. Troncati F, Paci M, Myftari T, Lombardi B (2013) Extracorporeal shock wave therapy reduces upper limb spasticity and improves motricity in patients with chronic hemiplegia: a case series. NeuroRehabilitation 33(3):399-405. https://doi.org/10.3233/NRE-130970

37. Deschuytere J, Dekeyser C, Deschuttere M, Rosselle N (1983) H reflexes in muscles of the lower and upper limbs in man: identification and clinical significance. Adv Neurol 39(9):51-60 
38. Powers RK, Campbell DL, Rymer WZ (1989) Stretch reflex dynamics in spastic elbow flexor muscles. Ann Neurol 25(1):32-42. https://doi.org/10.1 002/ana.410250106

39. Daliri SS, Forogh B, Emami Razavi SZ, Ahadi T, Madjlesi F, Ansari NN (2015) A single blind, clinical trial to investigate the effects of a single session extracorporeal shock wave therapy on wrist flexor spasticity after stroke. NeuroRehabilitation 36(1):67-72. https://doi.org/10.3233/NRE-141193

40. Sohn MK, Cho KH, Kim YJ, Hwang SL (2011) Spasticity and electrophysiologic changes after extracorporeal shock wave therapy on gastrocnemius. Ann Rehabil Med 35(5):599-604. https://doi.org/10.5535/a rm.2011.35.5.599

41. Marinelli L, Mori L, Solaro C, Uccelli A, Pelosin E, Currà A, Molfetta L, Abbruzzese G, Trompetto C (2014) Effect of radial shock wave therapy on pain and muscle hypertonia: a double-blind study in patients with multiple sclerosis. Mult Scler 21:622-629

\section{Publisher's Note}

Springer Nature remains neutral with regard to jurisdictional claims in published maps and institutional affiliations.

\section{Submit your manuscript to a SpringerOpen ${ }^{\circ}$ journal and benefit from:}

- Convenient online submission

- Rigorous peer review

- Open access: articles freely available online

High visibility within the field

- Retaining the copyright to your article

Submit your next manuscript at $\boldsymbol{\nabla}$ springeropen.com 